\title{
A p38 Mitogen-Activated Protein Kinase Inhibitor Arrests Active Alveolar Bone Loss in a Rat Periodontitis Model
}

Jill E. Rogers, * Fei Li, * Derek D. Coatney, * Jodie Otremba, * Jaclynn M. Kriegl, ${ }^{\dagger}$

Andrew A. Protter, ${ }^{\ddagger}$ Linda S. Higgins, $§$ Satyanarayana Medicherla, $\|$ and Keith L. Kirkwood*

Background: Gram-negative bacterial species, such as Actinobacillus actinomycetemcomitans, contain lipopolysaccharide (LPS) that initiates the innate immune system, resulting in inflammatory alveolar bone loss. LPS activates Toll-like receptors on membrane surfaces, stimulating many intracellular signaling cascades, including the p38 mitogen-activated protein kinase (MAPK). Activation of p38 signaling mediates inflammatory cytokine expression, contributing toward osteoclastogenesis and bone loss. The aim of this study was to determine whether the novel, orally active p38 MAPK inhibitor SD282 could arrest progression of LPS-induced alveolar bone destruction in rats.

Methods: Three groups of female Sprague-Dawley rats received LPS injections to the palatal molar gingiva three times per week for 4 weeks to establish periodontitis. From weeks 5 through 8, two groups received the drug SD282 $(\mathrm{N}=14)$ or $1 \%$ polyethylene glycol drug vehicle $(N=14)$ via oral gavage in addition to LPS injections. The third group continued to receive only LPS injections $(\mathrm{N}=8)$. Microcomputed tomography was used to measure volumetric alveolar bone loss, expressed as bone volume fraction (BVF). Expression of interleukin (IL) -1 and -6 and tumor necrosis factor-alpha (TNF- $\alpha$ ) was assessed by immunohistochemistry, and osteoclasts were enumerated by tartrate-resistant acid phosphatase staining.

Results: By 4 weeks, severe alveolar bone resorption was seen in LPS-injected animals. Administration of SD282 significantly blocked additional volumetric bone loss in the LPS-only versus LPS + SD282 groups $(0.37 \pm 0.01 \mathrm{BVF}$ versus $0.43 \pm 0.01 \mathrm{BVF} ; P<0.01)$. Significant reductions in IL-1 $\beta(P<0.01)$, TNF- $\alpha(P<0.05)$, and osteoclast formation $(P<0.01)$ occurred in the presence of SD282.

Conclusions: An orally active p38 MAPK inhibitor reduced LPS-induced inflammatory cytokine expression, osteoclastogenesis, and alveolar bone loss in rats. Within the limits of the current study, SD282 arrested periodontal disease progression, thus highlighting the therapeutic potential of this novel class of inhibitors. J Periodontol 2007; 78:1992-1998.

\section{KEY WORDS}

Alveolar bone loss; cytokines; lipopolysaccharide; mitogen-activated protein kinase; periodontitis; p38 inhibitor.

\footnotetext{
* Department of Periodontics and Oral Medicine, School of Dentistry, University of Michigan, Ann Arbor, MI.

$\dagger$ Orthopedics Research Laboratory, University of Michigan.

‡ Currently, Medivation, San Franscisco, CA; previously, Scios, Fremont, CA.

$\S$ Currently, InteKrin Therapeutics, Los Altos, CA; previously, Scios.

\|| Currently, Schering-Plough, Kenilworth, NJ; previously, Scios.
}

$\mathrm{P}$ eriopathogenic bacteria, including Actinobacillus actinomycetemcomitans, contain multiple virulence factors, such as lipopolysaccharide (LPS), that can activate the host inflammatory response. An improper or exuberate immune response leads to overproduction of inflammatory cytokines and, consequently, periodontal attachment loss and alveolar bone loss. ${ }^{1}$

Within periodontal tissues and gingival crevicular fluid, activated monocytes, macrophages, and fibroblasts produce interleukin (IL) $-1 \beta$, IL-6, and tumor necrosis factor-alpha $(\mathrm{TNF}-\alpha)$. $^{2-5}$ These cytokines synergistically stimulate the production of other inflammatory cytokines, matrix metalloproteinases (MMPs), and prostanoids. ${ }^{1,6}$

LPS increases osteoblastic expression of IL- $1 \beta$, TNF- $\alpha$, prostaglandin $\mathrm{E}_{2}\left(\mathrm{PGE}_{2}\right)$, and receptor activator of nuclear factor-kappa $B$ ligand (RANKL), which induces osteoclast activity, viability, and differentiation. ${ }^{7,8}$ Osteoclasts are activated further in the presence of A. actinomycetemcomitans by $\mathrm{CD} 4^{+} \mathrm{T}$ cells that have an increased expression of RANKL. 9,10

Mitogen-activated protein $\mathrm{ki}$ nases (MAPKs) are intracellular 
kinases required, in part, for inflammation and inflammatory bone loss through activation of inflammatory cytokines and MMPs. LPS binds to its appropriate membrane receptor (CD14 or Toll-like receptor-4), and triggers intracellular signaling cascades, inducing phosphorylation of multiple MAPKs within resident periodontal cell types. Targeted activation of p38 induced TNF- $\alpha$ and IL-1, -6 , and -8 via direct activation of gene transcription or mRNA stabilization. ${ }^{11-16}$ p38 MAPK also controls the synthesis of other compounds, including chemokines, metalloproteinases, prostaglandins, and nuclear factor-kappa B. ${ }^{17}$ In addition, our group recently demonstrated that IL-1 $\beta$ and TNF- $\alpha$-induced RANKL expression in bone marrow stromal cells requires p38 signaling for maximal expression, resulting in decreased induction of osteoclastogenesis in vitro. ${ }^{16}$

Therefore, blocking p38 activation could decrease inflammatory cytokine production and its deleterious effects. The p38 inhibitors SC409 and SD282 reduced and reversed bone and cartilage destruction in an experimental arthritis model. ${ }^{18,19}$ SD282 also was capable of preventing alveolar bone loss in a rat periodontitis model. ${ }^{20}$ The aim of the present study was to determine whether the orally active p38 inhibitor SD282 could stop the progression of alveolar bone loss initiated by $A$. actinomycetemcomitans LPS in an experimental rat model.

\section{MATERIALS AND METHODS}

\section{Animals}

Adult female Sprague-Dawley rats ${ }^{\text {Il }}$ were housed in pairs under specific pathogen-free conditions with food and tap water ad libitum. Once weekly, animals were weighed to ensure proper growth and nutrition. All protocols were approved by the University Committee on the Use and Care of Animals, University of Michigan.

\section{Bone Loss Induction and Administration of p38 Inhibitor}

Within tissue macrophages and other periodontal cells, MAPKs are activated by A. actinomycetemcomitans LPS. ${ }^{14}$ Inflammatory bone loss was initiated according to the aggressive periodontitis model described previously. ${ }^{21}$ LPS was extracted from A. actinomycetemcomitans strain Y4 (serotype B) and purified, containing $<0.001 \%$ nucleic acid by spectrophotometry and $0.7 \%$ protein by bicinchoninic acid protein assay. Three groups of animals received LPS injections. Using a 33-gauge Hamilton syringe, $2 \mu \mathrm{l}$ LPS was injected directly into the palatal interproximal gingiva mesial and distal to the second molar bilaterally. This procedure was repeated three times each week for 8 weeks. To facilitate LPS injections, anesthesia was induced with $4 \%$ to $5 \%$ isoflurane and maintained with $1 \%$ to $2 \%$ isoflurane.
The p38 inhibitor SD282 and its vehicle, 1\% polyethylene glycol, were administered via oral gavage. With this technique, liquid compounds can be administered directly into the stomach of experimental animals. A bulb-tipped gastric gavage needle was passed through the mouth and into the esophagus. The opposite end of the needle was attached to a syringe containing the desired substance. This is a systemic administration that ensures the entire volume of fluid is delivered. All animal handlers received proper orientation and training in this method prior to initiation of the experiment. Beginning the fifth week, the test group $(\mathrm{N}=14)$ received twice daily oral gavage of SD282 $(45 \mathrm{mg} / \mathrm{kg})$, whereas the control group $(\mathrm{N}=14)$ received twice daily oral gavage of drug vehicle. The third group $(\mathrm{N}=8)$ continued to receive LPS injections only until the end of the experiment. At 8 weeks, all animals were sacrificed by carbon dioxide asphyxiation. Maxillae were hemisected, and posterior block sections were immersed directly in $10 \%$ buffered formalin fixative solution for $\geq 72$ hours.

\section{Microcomputed Tomography}

Non-demineralized rat maxillae were scanned in $70 \%$ ethanol by a cone-beam microcomputed tomography $(\mu \mathrm{CT})$ system. ${ }^{*}$ Scans were reconstructed at a mesh size of $18 \mu \mathrm{m}^{3}$ to create a three-dimensional digitized image of each specimen. Computer software** was used to analyze the images, set to a threshold of 1,621 and a surface quality factor of 0.5 . To create a standardized orientation, the buccal and palatal plates were aligned horizontally with the $\mathrm{x}$ axis, and the $\mathrm{x}$ plane was made to cross through the palatal roots.

The amount of bone in each specimen was assessed volumetrically by an independent, masked examiner, according to methods described recently. ${ }^{20,21}$ Volumetric changes were assessed within a region of interest (ROI), the parameters of which were dictated by the dental anatomy of the specimens. The mesialdistal width of the ROI was set at the interproximal height of contour of the molars, whereas the height of the ROI was equal to the distance from molar cusp tips to root apices. ROI depth was determined by the bucco-lingual dimension of the teeth plus 100 voxels $\left(1.8 \mathrm{~mm}^{3}\right)$. To express the amount of bone present, the bone volume fraction (BVF) was calculated as follows: $\mathrm{BVF}=$ amount of remaining bone in $\mathrm{ROI} \div \mathrm{ROI}$.

\section{Immunohistochemistry and Tartrate-Resistant Acid Phosphatase Staining}

Formalin-fixed maxillae were decalcified in $10 \%$ EDTA solution (replenished three times per week) at $4^{\circ} \mathrm{C}$ for 2 weeks. The maxillae were embedded in

\footnotetext{
I Charles River, Wilmington, MA.

\# MicroView 2.1.2, GE Healthcare BioSciences, Chalfont St. Giles, U.K

** MicroView 2.1.2, GE Healthcare BioSciences.
} 
paraffin, and 5- $\mu \mathrm{m}$ sections were prepared. Some specimens from each group were selected for standard hematoxylin and eosin staining. The remainder was used for immunohistochemistry. First, deparaffinized tissue sections were placed in antigen retrieval buffer $^{\dagger \dagger}$ in a pressure chamber ${ }^{\dagger \dagger}$ for 15 minutes. After cooling the specimens, primary antibodies for antirat IL-6, anti-rat IL-1 $\beta$, and anti-rat TNF- $\alpha$ monoclonal antibodies were added (1:200 each). $\S \S$ Cytokine

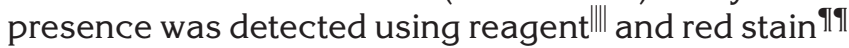
according to the manufacturer's instructions. Control sections were incubated with preimmunoserum to assess background staining level. Photographs of all prepared sections were taken using an inverted microscope $^{\# \#}$ and digital camera. ${ }^{* * *}$

Cytokine expression was quantified as described recently. ${ }^{20,21}$ The overall intensity of red stain on each slide was judged by three independent, calibrated, masked examiners. Examiners were calibrated by comparing digitized images of a known color intensity to a standardized score sheet showing examples of each intensity level. Scores of 1 represented $<20 \%$ positive stain, 2 represented $21 \%$ to $40 \%$ positive stain, 3 represented $41 \%$ to $60 \%$ positive stain, and 4 represented $>60 \%$ positive stain. For data collection, slides were displayed in random order and lacked group identifying information. Slides were differentiated by an assigned number, the legend for which was concealed from the examiners.

The presence of osteoclasts was detected by tartrate-resistant acid phosphatase (TRAP) staining. ${ }^{\dagger \dagger}$ Active osteoclasts, defined as multinucleated TRAPpositive cells in contact with the bone surface, were counted per slide by one examiner at $60 \times$ magnification. Slides from approximately the same sagittal sections were used, and slides were identified by assigned number, as for immunostaining.

\section{Statistical Analysis}

The minimum sample size for detecting a difference of $50 \%$ between groups was six animals per group. ${ }^{22,23}$ This was calculated considering a power of $80 \%$. Results were expressed as means for the entire group. One maxilla section per animal contributed one measured value toward the mean for its assigned group. Statistical comparison was conducted using commercially available software. ${ }^{\neq} \neq \neq$Differences among data were found by one-way analysis of variance, followed by Bonferroni multiple comparison test. $P$ values $<0.05$ were considered statistically significant.

\section{RESULTS}

\section{Animals}

Three animals were lost in the LPS + vehicle and two in the LPS + SD282 groups. Although the exact causes of death were unknown, one explanation could be the oral gavage technique. Asphyxiation is possible if the needle is placed incorrectly and liquid is administered into the lungs instead of the stomach. Oral administration of high doses of SD282 for 8 weeks had no significant biologic effects in rats. ${ }^{20}$

\section{SD282 Maintains Alveolar Bone}

To evaluate the extent of alveolar bone loss and periodontal destruction, $\mu \mathrm{CT}$ was used as described by Park et al. ${ }^{24}$ In the control and experimental groups, horizontal bone loss with furcation involvement was evident. This bone loss was most severe between the molars (Fig. 1), but also was evident at the second molar palatal furcation.

Volumetric measurements of alveolar bone revealed a significant therapeutic effect from the p38 inhibitor (Fig. 2). Continued LPS injections decreased the BVF between 4 and 8 weeks, from $0.40 \pm 0.01$ to $0.37 \pm 0.01$. However, the administration of SD282 reversed this process and increased the BVF to $0.43 \pm$ 0.01 . The difference between the 8-week LPS and 8 -week LPS with SD282 was significant at $P<0.01$. These data strongly suggested that the SD282 arrested further progression of the LPS-induced bone loss and suggested reversal of LPS-induced bone loss in this rat model.

\section{SD282 Decreases Inflammatory Cytokine Expression}

Levels of IL- $1 \beta$ and -6 and TNF- $\alpha$ were assessed via scoring of immunohistochemically stained slides by calibrated, masked, and independent examiners. Some slides had to be omitted because of high levels of background staining that made detection of color differences difficult. Overall, cytokine levels were decreased in the presence of the p38 inhibitor SD282 (Fig. 3). This decrease was significant for IL-1 $\beta$ ( $P=$ $0.0019)$ and TNF- $\alpha(P=0.0193)$, but approached significance for IL-6 $(P=0.0726)$. For comparison, animals not injected with LPS showed cytokine levels $<1$. This was seen in a similar experiment in which rats were given SD282 alone by oral gavage. ${ }^{20}$

\section{SD282 Inhibits Osteoclastogenesis}

Osteoclastogenesis, a prerequisite for inflammatory osteolysis, was measured by the number of TRAPpositive multinucleated cells per sample (Fig. 4). There were significantly fewer osteoclasts present in the LPS + SD282 versus the 8-week LPS groups $(P<0.01)$. The addition of the $\mathrm{p} 38$ inhibitor decreased

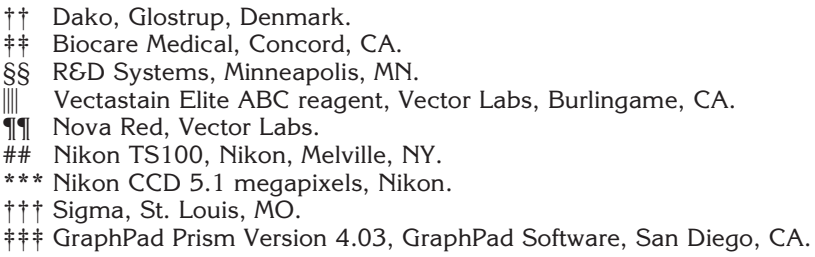



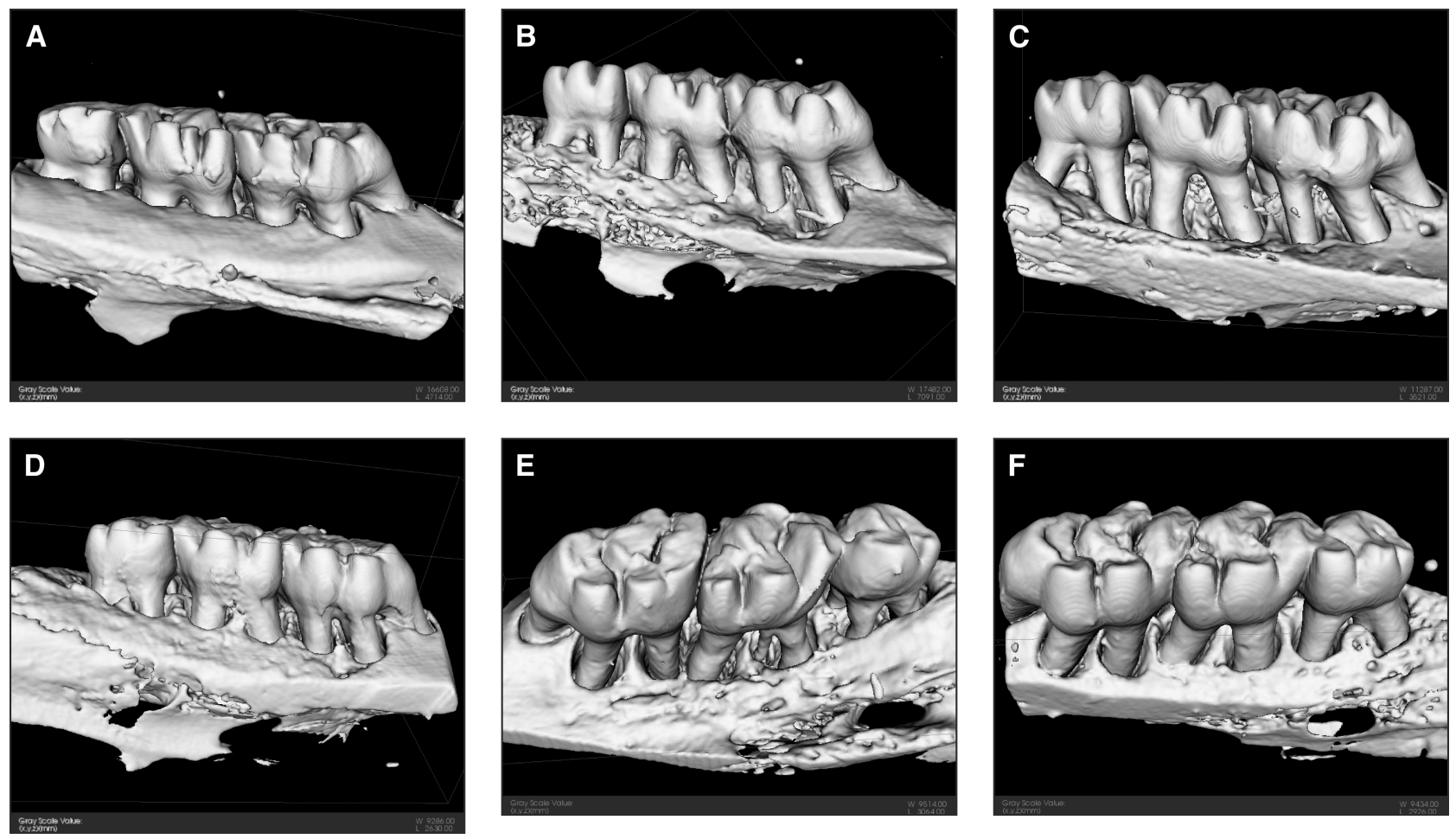

Figure I.

$\mu C T$ shows that SD282 limits progression of established LPS-induced alveolar bone loss. Selected three-dimensional isoform displays of rat maxillae show: (A) normal bone levels in a non-LPS injected animal, (B) interproximal and palatal bone loss after 8 weeks of LPS, (C) interproximal and palatal bone loss after 4 weeks of LPS followed by 4 weeks of LPS + vehicle, and (D) maintenance of bone after 4 weeks of LPS followed by 4 weeks of LPS + SD282. Additional isoforms from the LPS-only group at 4 weeks $(\mathbf{E})$ and LPS + SD282 group at 8 weeks (F) allow comparison between baseline for drug administration and final therapeutic outcome.

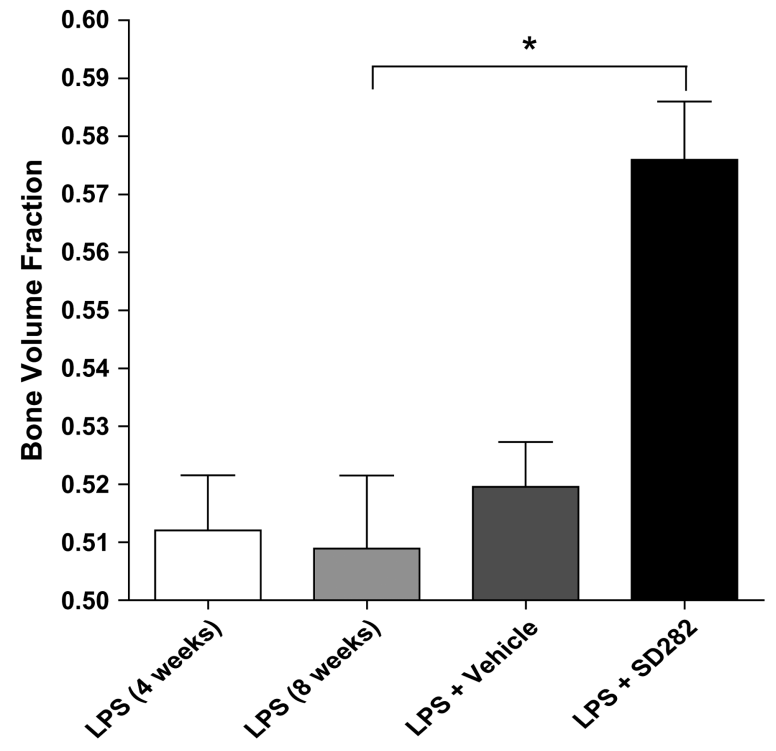

Figure 2.

SD282 maintains alveolar bone. Bone volume within an ROI as assessed by $\mu C T$ software. Data are presented as fraction of bone within the ROI (teeth are constant between samples). The difference between the 8-week LPS $(N=8)$ and LPS + SD282 $(N=12)$ groups was significant ( $* P<0.00$ I). The difference was $P<0.00$ I for the LPS + SD282 and 4-week LPS and LPS + vehicle groups.

osteoclast levels below baseline (4 weeks of LPS injection).

\section{DISCUSSION}

The current study investigated the therapeutic potential of a novel orally active p38 inhibitor, SD282, in arresting periodontitis in vivo. Inflammatory alveolar bone loss was established in rats by local injection of periopathogenic LPS for 4 weeks, then SD282 was given via oral gavage (along with continued LPS injections) for an additional 4 weeks. Assessment of volumetric bone loss, inflammatory cytokine expression, and osteoclast formation revealed that SD282 was effective at reducing osteolysis.

Previously, our research group showed that the $A$. actinomycetemcomitans LPS injection method is capable of producing severe alveolar bone loss in rats within 8 weeks. ${ }^{21}$ Compared to saline-injected controls, LPS-injected animals exhibited significantly greater linear bone loss (1.04 versus $0.40 \mathrm{~mm}$ cementoenamel junction-alveolar bone crest [CEJ-ABC]; $P<0.01)$ and volumetric bone loss $(0.37$ versus 0.42 BVF; $P<0.01)$. LPS-injected animals also had significantly increased levels of inflammatory cell 

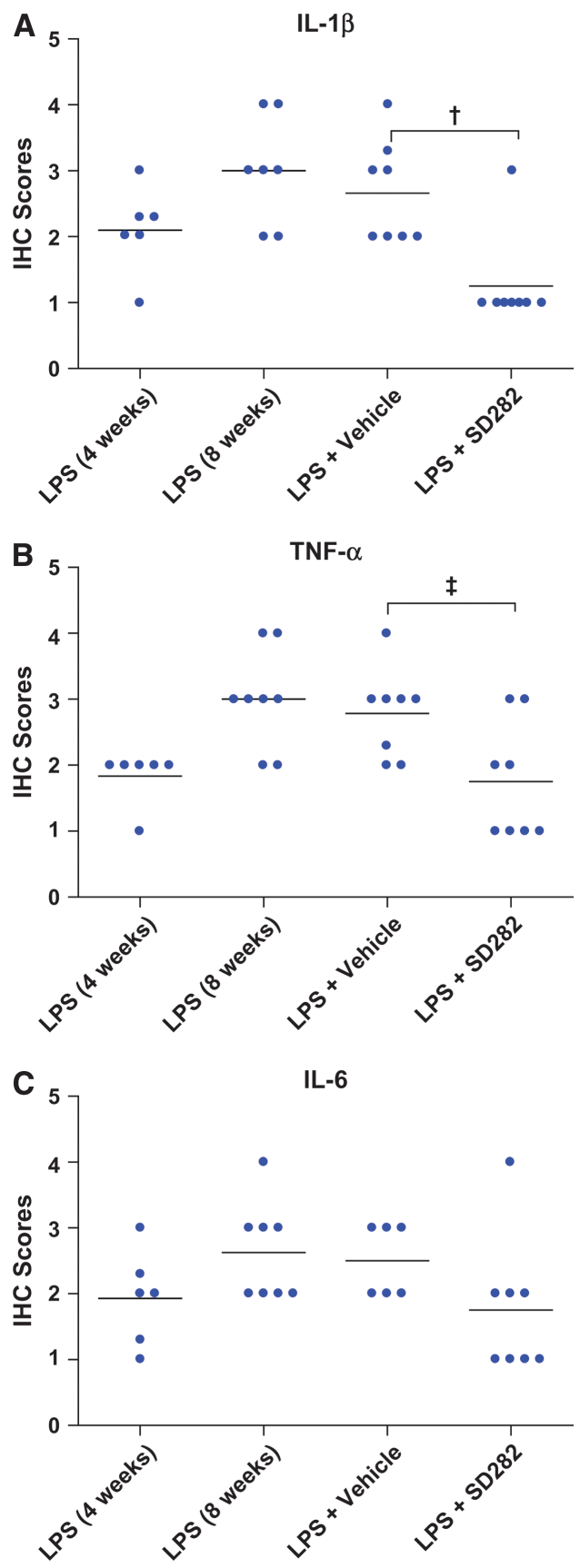

Figure 3.

SD282 decreases inflammatory cytokine expression. Immunohistochemistry (IHC) scores of 0 denote absence of stain, whereas scores of 4 indicate intense stain and high prevalence of cytokines. Significant differences in IL-I $\beta\left({ }^{\dagger} \mathrm{P}<0.01\right)$ and TNF- $\alpha$ $\left({ }^{\ddagger} \mathrm{P}<0.05\right)$ were seen between the 8 -week $L P S+$ vehicle $(N=11)$ and LPS + SD282 $(N=12)$ groups.

infiltrate, inflammatory cytokine (IL-1 $\beta$ and -6 and TNF- $\alpha$ ) expression, and osteoclastogenesis. ${ }^{21}$

This orally active p38 inhibitor is able to prevent inflammatory alveolar bone loss in vivo, as demonstrated recently by our laboratory. ${ }^{20}$ SD282 was administered concomitantly with LPS injection for 8 weeks. Immuno-

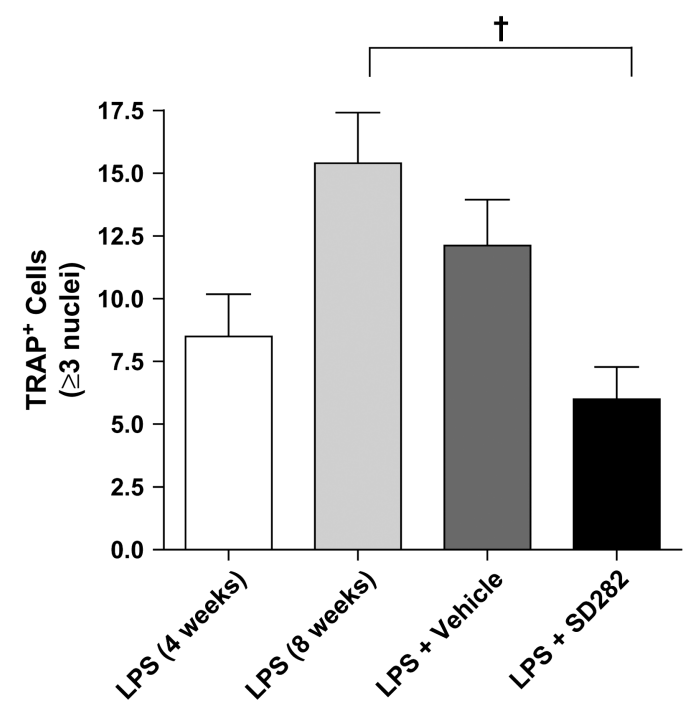

Figure 4.

SD282 inhibits osteoclast formation. Values represent the mean for all specimens from each group. A statistically significant reduction was seen between the 8-week LPS and LPS + SD282 groups ( ${ }^{\dagger} \mathrm{P}<0.01$ ).

histochemistry results showed that LPS increased phospho-p38 $\alpha$, whereas SD282 decreased active p38 $\alpha(P<0.001)$. Moreover, SD282 significantly protected against loss of bone height, area, and volume at low $(15 \mathrm{mg} / \mathrm{kg})$ and high doses $(45 \mathrm{mg} / \mathrm{kg})$.

In the current study, changes in bone quantity and quality were apparent on the physical specimens and $\mu \mathrm{CT}$ scans. Alterations in bone quality were striking, with LPS-only specimens exhibiting "shelled-out" or "Swiss cheese" bone. Up to 4 weeks, the resorption was horizontal; however, after 4 weeks, the bone loss was mainly within cancellous bone. Hypothetical explanations for this include needle penetration through the cortical plate, injection into the periodontal ligament space, or a local cytokine-driven process within the marrow space. This last theory is supported by the fact that p38 MAPK is required for TNF- $\alpha-$ and IL- $1 \beta-$ induced RANKL expression in bone marrow stromal cells. ${ }^{16}$ Some studies have shown contrasting resorption patterns between cortical and cancellous bone. For instance, active vitamin D metabolites act on mature osteoblasts to increase osteoprotegerin. In mice, inhibition of these metabolites was less effective in cancellous bone, rendering it susceptible to resorption. ${ }^{25}$ Thus, a volumetric assessment of bone level seemed most appropriate.

When analyzing the results of this study, it is important to remember that the drug was administered in a model of preexisting active disease. In such a model, the true baseline is a point at which bone loss or disease already has been established. Therefore, the therapeutic goal is to prevent further advancement of the disease. 
This is analogous to a clinical situation where active periodontitis has resulted in clinically evident alveolar bone loss. The contemporary concept of periodontal therapy focuses on eliminating bacteria through mechanical means and chemotherapeutics. However, none of these methods has proven universally efficacious, particularly in the case of tissue-invasive species such as A. actinomycetemcomitans. ${ }^{26}$ Moreover, patients' compliance with oral hygiene instruction and maintenance recalls are extremely influential on treatment outcomes. ${ }^{27}$ Therefore, the concept of host modulation has garnered attention in periodontal research. Studies showed significant clinical improvement and reduction of alveolar bone destruction by modulating arachidonic acid metabolites 28,29 and MMPs. ${ }^{30,31}$ Successful attempts have been made to alter osteoclast activity through bisphosphonates ${ }^{32,33}$ and a novel vacuolar adenosine triphosphatase. ${ }^{34}$ However, these therapies target singular mechanisms of alveolar bone destruction.

Conversely, p38 MAPK is an upstream effector common to many inflammatory cytokines. Activated monocytes, macrophages, and fibroblasts in the periodontium produce cytokines and prostanoids, including TNF- $\alpha$, IL- $1 \beta$, and -6 , and PGE ${ }_{2}{ }^{2}$ These cytokines induce the production of several inflammatory enzymes and mediators, such as MMPs, prostaglandins, and RANKL. ${ }^{35,36}$ For instance, TNF- $\alpha$ and IL-1 activate bone marrow stromal cells and macrophages to induce osteoclastogenesis through RANK-RANKL interaction. ${ }^{37}$ Inhibition of IL- 1 and TNF- $\alpha$ decreases the spread of inflammatory infiltrate and loss of connective tissue attachment and alveolar bone. ${ }^{36,38}$ Because these cytokines act synergistically, simultaneous blockage of both is substantially more effective than blocking one. ${ }^{39}$ In the first human study, ${ }^{40}$ a single dose of p38 inhibitor decreased TNF- $\alpha$ and IL- 1 and -6 levels by $90 \%$.

In this study, SD282 had a slight anabolic effect; the difference between the 8-week LPS-only and LPS + SD282 groups was significant for increased bone volume. The reasons for this are unclear, but it may due to a relatively high suppression of osteoclastogenesis without compensatory shut-off of osteoblastic differentiation. However, it is not believed that osteoclastogenesis is eliminated completely by p38 inhibition. Systemically, a number of hormones and cytokines modulate osteoclastogenesis: parathyroid hormone (PTH); calcitriol; PTH-related protein (PTHrP); $\mathrm{PGE}_{2}$; and IL-1 $\beta,-6$, and IL-11. ${ }^{1}$ Of these, PTH and PTHrP can activate osteoclastogenesis independent of p38 signaling. ${ }^{41}$ Conceptually, this makes p38 inhibitor strategies appealing as a host-modulating agent for treatment of periodontitis because physiologic bone turnover (induced by PTH/PTHrP) would occur, but inflammatory bone loss (induced by LPS, IL-1 $\beta$, and TNF- $\alpha$ ) would be pharmacologically antagonized.

\section{CONCLUSIONS}

Treatment with an orally active p38 MAPK inhibitor arrested established periodontal disease progression in vivo. Specifically, SD282 reduced LPS-induced inflammatory cytokine expression, osteoclastogenesis, and alveolar bone loss in rats. Results such as these support further development of this novel class of therapeutics and their potential role in treating inflammatory diseases, such as periodontitis, in humans.

\section{ACKNOWLEDGMENTS}

The authors thank Paul Bronson, research associate, State University of New York at Buffalo, Buffalo, New York, for his technical support in LPS preparation and Dr. Steven Goldstein, director, University of Michigan Orthopaedic Research Laboratories, University of Michigan Core Center for Musculoskeletal Disorders. This study was supported by Scios, the Department of Defense (grant W81XWH-05-0075 to Dr. Kirkwood), and the National Institutes of Health (grant P30AR46024). At the time this study was conducted, Andrew A. Protter, vice president of biology; Linda S. Higgins, senior director of research; and Satyanarayana Medicherla, staff scientist, were employed by Scios.

\section{REFERENCES}

1. Kirkwood KL, Cirelli J, Rogers JE, Giannobile WV. Novel host response therapeutic approaches to treat periodontal diseases. Periodontol 2000 2007;43:294-315.

2. Lee HJ, Kang IK, Chung CP, Choi SM. The subgingival microflora and gingival crevicular fluid cytokines in refractory periodontitis. J Clin Periodontol 1995;22:885-890.

3. Leng SX, Elias JA. Interleukin-11 inhibits macrophage interleukin-12 production. J Immunol 1997;159:2161 2168.

4. Chen CC, Chang KL, Huang JF, Huang JS, Tsai CC. Interleukin- 6 production by human gingival fibroblasts following stimulation with Actinobacillus actinomycetemcomitans (in Chinese). Kaohsiung J Med Sci 1998; 14:367-378.

5. Tsai CC, Ho YP, Chen CC. Levels of interleukin-1 beta and interleukin-8 in gingival crevicular fluids in adult periodontitis. J Periodontol 1995;66:852-859.

6. Haffajee AD, Socransky SS. Microbial etiological agents of destructive periodontal diseases. Periodontol 2000 1994;5:78-111.

7. Roux S, Orcel P. Bone loss. Factors that regulate osteoclast differentiation: An update. Arthritis Res 2000; 2:451-456.

8. Suda T, Kobayashi K, Jimi E, Udagawa N, Takahashi N. The molecular basis of osteoclast differentiation and activation. Novartis Found Symp 2001;232:235-247.

9. Mahamed DA, Marleau A, Alnaeeli M, et al. G(-) anaerobes-reactive CD4+ T-cells trigger RANKLmediated enhanced alveolar bone loss in diabetic NOD mice. Diabetes 2005;54:1477-1486.

10. Teng YT, Nguyen H, Gao X, et al. Functional human $\mathrm{T}$-cell immunity and osteoprotegerin ligand control alveolar bone destruction in periodontal infection. J Clin Invest 2000;106:R59-R67.

11. Adams JL, Badger AM, Kumar S, Lee JC. p38 MAP kinase: Molecular target for the inhibition of 
pro-inflammatory cytokines. Prog Med Chem 2001; 38:1-60

12. Hoffmann E, Dittrich-Breiholz O, Holtmann H, Kracht M. Multiple control of interleukin-8 gene expression. J Leukoc Biol 2002;72:847-855.

13. Kirkwood K, Martin T, Andreadis ST, Kim YJ. Chemically modified tetracyclines selectively inhibit IL-6 expression in osteoblasts by decreasing mRNA stability. Biochem Pharmacol 2003;66:1809-1819.

14. Patil C, Rossa CJ, Kirkwood K. Actinobacillus actinomycetemcomitans lipopolysaccharide induces interleukin- 6 expression through multiple mitogen-activated protein kinase pathways in periodontal ligament fibroblasts. Oral Microbiol Immunol 2006;21:392-398.

15. Patil C, Zhu X, Rossa C Jr., Kim YJ, Kirkwood KL. p38 MAPK regulates IL-1beta induced IL- 6 expression through mRNA stability in osteoblasts. Immunol Invest 2004;33:213-233.

16. Rossa C, Ehmann K, Liu M, Patil C, Kirkwood KL. MKK3/6-p38 MAPK signaling is required for IL-1beta and TNF-alpha-induced RANKL expression in bone marrow stromal cells. J Interferon Cytokine Res 2006; 26:719-729.

17. Lee JC, Kumar S, Griswold DE, Underwood DC, Votta BJ, Adams JL. Inhibition of p38 MAP kinase as a therapeutic strategy. Immunopharmacology 2000;47: 185-201.

18. Mbalaviele G, Anderson G, Jones A, et al. Inhibition of p38 mitogen-activated protein kinase prevents inflammatory bone destruction. J Pharmacol Exp Ther 2006; 317:1044-1053.

19. Medicherla S, Ma JY, Mangadu R, et al. A selective p38 alpha mitogen-activated protein kinase inhibitor reverses cartilage and bone destruction in mice with collagen-induced arthritis. J Pharmacol Exp Ther 2006; 318:132-141.

20. Kirkwood KL, Li F, Rogers JE, et al. A p38-alpha selective mitogen activated protein kinase inhibitor prevents periodontal bone loss. J Pharmacol Exp Ther 2007;320:56-63.

21. Rogers JE, Li F, Coatney DD, et al. Actinobacillus actinomycetemcomitans lipopolysaccharide-mediated experimental bone loss model for aggressive periodontitis. J Periodontol 2007;78:550-558.

22. Guo L, Lim KB, Gunn JS, et al. Regulation of lipid A modifications by Salmonella typhimurium virulence genes phoP-phoQ. Science 1997;276:250-253.

23. Karimbux NY, Ramamurthy NS, Golub LM, Nishimura I. The expression of collagen I and XII mRNAs in Porphyromonas gingivalis-induced periodontitis in rats: The effect of doxycycline and chemically modified tetracycline. J Periodontol 1998;69:34-40.

24. Park C, Abramson Z, Taba M, et al. Three-dimensional micro-computed tomographic imaging of alveolar bone in experimental bone loss or repair. $J$ Periodontol 2007;78:273-281.

25. Baldock PA, Thomas GP, Hodge JM, et al. Vitamin D action and regulation of bone remodeling: Suppression of osteoclastogenesis by the mature osteoblast. J Bone Miner Res 2006;21:1618-1626.

26. Christersson LA, Zambon JJ. Suppression of subgingival Actinobacillus actinomycetemcomitans in localized juvenile periodontitis by systemic tetracycline. J Clin Periodontol 1993;20:395-401.

27. Wilson TG Jr. How patient compliance to suggested oral hygiene and maintenance affect periodontal therapy. Dent Clin North Am 1998;42:389-403.
28. Jeffcoat MK, Williams RC, Reddy MS, English R, Goldhaber P. Flurbiprofen treatment of human periodontitis: Effect on alveolar bone height and metabolism. J Periodontal Res 1988;23:381-385.

29. Williams RC, Jeffcoat MK, Howell TH, et al. Altering the progression of human alveolar bone loss with the non-steroidal anti-inflammatory drug flurbiprofen. $J$ Periodontol 1989;60:485-490.

30. Caton JG, Ciancio SG, Blieden TM, et al. Treatment with subantimicrobial dose doxycycline improves the efficacy of scaling and root planing in patients with adult periodontitis. J Periodontol 2000;71:521-532.

31. Ciancio S, Ashley R. Safety and efficacy of subantimicrobial-dose doxycycline therapy in patients with adult periodontitis. Adv Dent Res 1998;12:27-31.

32. Reddy MS, Weatherford TW 3rd, Smith CA, West BD, Jeffcoat MK, Jacks TM. Alendronate treatment of naturally-occurring periodontitis in beagle dogs. $J$ Periodontol 1995;66:211-217.

33. Rocha M, Nava LE, Vazquez de la Torre C, SanchezMarin F, Garay-Sevilla ME, Malacara JM. Clinical and radiological improvement of periodontal disease in patients with type 2 diabetes mellitus treated with alendronate: A randomized, placebo-controlled trial. J Periodontol 2001;72:204-209.

34. Niikura K. Vacuolar ATPase as a drug discovery target. Drug News Perspect 2006;19:139-144.

35. Assuma R, Oates T, Cochran D, Amar S, Graves DT. IL-1 and TNF antagonists inhibit the inflammatory response and bone loss in experimental periodontitis. J Immunol 1998;160:403-409.

36. Graves DT, Delima AJ, Assuma R, Amar S, Oates T, Cochran D. Interleukin-1 and tumor necrosis factor antagonists inhibit the progression of inflammatory cell infiltration toward alveolar bone in experimental periodontitis. J Periodontol 1998;69:1419-1425.

37. Wei S, Kitaura H, Zhou P, Ross FP, Teitelbaum SL. IL-1 mediates TNF-induced osteoclastogenesis. J Clin Invest 2005;115:282-290.

38. Delima AJ, Oates T, Assuma R, et al. Soluble antagonists to interleukin-1 (IL-1) and tumor necrosis factor (TNF) inhibits loss of tissue attachment in experimental periodontitis. J Clin Periodontol 2001;28:233-240.

39. Zwerina J, Hayer S, Tohidast-Akrad M, et al. Single and combined inhibition of tumor necrosis factor, interleukin-1, and RANKL pathways in tumor necrosis factor-induced arthritis: Effects on synovial inflammation, bone erosion, and cartilage destruction. Arthritis Rheum 2004;50:277-290.

40. Parasrampuria DA, de Boer P, Desai-Krieger D, Chow AT, Jones CR. Single-dose pharmacokinetics and pharmacodynamics of RWJ 67657, a specific p38 mitogen-activated protein kinase inhibitor: A first-inhuman study. J Clin Pharmacol 2003;43:406-413.

41. Fu Q, Jilka R, Manolagas S, O'Brien C. Parathyroid hormone stimulates receptor activator of NFkappa B ligand and inhibits osteoprotegerin expression via protein kinase A activation of cAMP-response elementbinding protein. J Biol Chem 2002;277:48868-48875.

Correspondence: Dr. Keith L. Kirkwood, Department of Periodontics and Oral Medicine, University of Michigan, 3349 Dental, 1011 N. University Ave., Ann Arbor, MI 481091078. Fax: 734/763-5503; e-mail: klkirk@umich.edu.

Submitted February 20, 2007; accepted for publication May 2, 2007. 